\title{
El análisis de la colección 0 . Menghin del Instituto de Arqueología (Facultad de Filosofía y Letras, Universidad de Buenos Aires). Artefactos líticos provenientes de la costa rionegrina
}

\author{
Jimena Alberti \\ Recibido 19 de octubre 2018. Aceptado 14 de junio 2019
}

\begin{abstract}
RESUMEN
En este trabajo se presentan los resultados del análisis tecnomorfológico y de materias primas de la colección de materiales líticos recolectada por Oswald Menghin entre los años 1951 y 1955 en la costa rionegrina del golfo San Matías. Dicho conjunto de materiales se encuentra depositado en el Instituto de Arqueología de la Facultad de Filosofía y Letras de la Universidad de Buenos Aires y está integrado, además de por los materiales líticos, por cerámica y valvas de moluscos. Aquí se presentan las tendencias generales de la muestra lítica y se realiza una breve comparación con los materiales del área recuperados por el equipo de investigación que actualmente trabaja allí. A partir de este estudio se pudieron ampliar ciertos aspectos del conocimiento acerca de los artefactos líticos manufacturados, utilizados y descartados por los grupos humanos que habitaron la costa rionegrina. Consideramos que el análisis de colecciones privadas, de museos y/o universidades es fundamental, ya que permite su puesta en valor científica y la generación de información que esté disponible para la comunidad en general.
\end{abstract}

Palabras clave: Golfo San Matías; Escuela Histórico-Cultural; Menghin; Conjuntos líticos.

\begin{abstract}
ANALYSIS OF THE O. MENGHIN LITHIC COLLECTION, INSTITUTE OF ARCHEOLOGY, FACULTAD DE FILOSOFÍA Y LETRAS, UNIVERSIDAD DE BUENOS AIRES. ARTIFACTS FROM RÍO NEGRO PROVINCE COAST. In this paper the results of the techno-morphological and raw material analyses of the collection of lithic materials collected by Oswald Menghin between 1952 and 1955 along the coast of San Matías Gulf (Río Negro province, Argentina) are presented. The collection is stored at the Institute of Archaeology, Facultad de Filosofía y Letras, Universidad de Buenos Aires. In addition to lithic artifacts, the collection contains ceramic sherds and mollusk shells. Patterns in the lithic material are presented and a brief comparison made with the archaeological remains recovered from the area by the research team. This study enlarged knowledge of aspects of lithic artifact manufacture, use and discard by the human groups that inhabited coastal Río Negro province. The analysis of private museums and/or university collections is considered essential since it highlights the scientific value of such collections and allows the generation of information that can be made available to the scientific community and general public.
\end{abstract}

Keywords: San Matías Gulf, Historical-Cultural School; Menghin; Lithic assamblages artifacts.

Jimena Alberti. Consejo Nacional de Investigaciones Científicas y Técnicas (CONICET). Instituto Multidisciplinario de Historia y Ciencias Humanas (IMHICIHU). Saavedra I5, 5to. piso ( I083), Buenos Aires, Argentina. E-mail: jimealberti@gmail.com 


\section{LOS ANÁLISIS DE COLECCIONES DE MATERIALES ARQUEOLÓGICOS}

El estudio de colecciones de materiales arqueológicos con información acerca de su procedencia -las cuales se encuentran habitualmente en manos de coleccionistas privados o depositadas en museos y/o universidades- es una actividad que ha tomado fuerza en los últimos años dentro de la arqueología. En el pasado, dichas colecciones contribuyeron al estudio del origen y el desarrollo de los grupos humanos a partir de descripciones de los objetos materiales que las formaban (Balesta y Zagorodny 2000). En la actualidad, hay una tendencia que implica su análisis a la luz de metodologías modernas para obtener nuevas fuentes de conocimiento. De acuerdo con Balesta y Zagorodny (2000), es necesario contextualizar las colecciones respondiendo preguntas acerca de cómo y cuándo se formó la colección y si existe algún registro de la recuperación de los materiales, entre otras. De acuerdo con Bernal et al. (2008), si estos conjuntos de materiales se encuentran bien referenciados, pueden aportar información muy valiosa a la investigación acerca de una temática determinada. En este sentido, Borrero sostiene que "la falta de contexto o cronología no constituye un 'criterio' de rechazo, sino de selección de la pertinencia de la muestra para realizar esfuerzos de investigación" (Borrero 2015: 9). En el caso de los artefactos líticos, por ejemplo, el análisis de colecciones permite ampliar el conocimiento acerca de la manufactura, uso y descarte de artefactos, de las zonas de aprovisionamiento de materias primas líticas en un área dada, del tipo de tecnología que fue utilizada, entre otros aspectos.

De acuerdo con Pérez de Micou (1998), existen en los museos dos tipos de colecciones: las casuales y las sistemáticas. Las primeras han sido desechadas por los investigadores debido a los métodos con los que habrían sido obtenidas, los cuales no se adecúan a los estándares científicos (Goetze y Mills 1991). Este tipo de recolección no implica necesariamente una falta de documentación de los materiales, sino un sesgo propio de la formación de la colección cuya orientación es necesario determinar.

Las denominadas colecciones sistemáticas, por otro lado, suelen estar formadas de manera organizada para incrementar el conocimiento antropológi$\mathrm{co}$, independientemente de si los recolectores fueron amateurs o profesionales. En estas colecciones hay una coherencia interna porque fueron reunidas para responder a algún problema antropológico en particular (Ford 1979; Parezo 1987) (lo cual también implica un sesgo), y suelen tener documentación respaldatoria que las acompaña. Según Pérez de Micou (1998), estos conjuntos pueden clasificarse en pasivos (donaciones) y activos, que son aquellos que fueron obtenidos por personal del museo, de manera directa o a partir de intermediarios.

Entonces, como ya se ha mencionado, el potencial de estudio de una colección está generalmente supeditado a que exista documentación probatoria de su origen y transporte hasta el lugar de depósito y a que sea accesible (Pérez de Micou 1998). Es necesario cuestionarse qué fue lo que se recolectó, qué no y por qué razón, y quién reunió los materiales, ya que generalmente sus preconceptos se ven reflejados allí. También es necesario entender cómo se almacena la colección, cómo es usada por parte de la institución que la guarda y cómo son los procesos de restauración y conservación.

Los estudios de colecciones se han desarrollado sobre diversos ítems del registro arqueológico. A modo de ejemplo, se pueden mencionar los trabajos de Buc y Coronel (2013) sobre los instrumentos óseos provenientes del humedal del Paraná inferior de la colección L. M. Torres, depositados en el Museo de Ciencias Naturales de La Plata; el de Bonomo y colaboradores, que analizaron los materiales líticos, óseos y cerámicos también de las colecciones del Museo de La Plata provenientes del delta del Paraná (Bonomo et al. 2009; Bonomo 2013); el de Alfonso-Durruty et al. (2015), donde se presenta el análisis de restos humanos depositado en el Centro de Estudios del Hombre Austral (Universidad de Magallanes) en Punta Arenas, Chile; el de Borrero y Borella (2010), quienes estudiaron los arpones óseos provenientes de Tierra del Fuego y del Estrecho de Magallanes, alojados en el Museo Etnográfico de la UBA y en el Museo Británico de Londres; el de Castro Esnal acerca del estudio de dos colecciones líticas depositadas en el Museo de América de Madrid (Castro Esnal 2015); el de Zilio y colaboradores, quienes analizaron la colección Pedro Dade, depositada en el Museo de La Plata y en museos de Dinamarca y Alemania (Zilio et al. 2018), entre otros. Finalmente, en la costa rionegrina, Borella y Buc (2009) y Marani y Cardillo (2010) analizaron los instrumentos óseos de una colección privada integrada por materiales provenientes de Saco Viejo, localidad arqueológica ubicada en la costa norte del golfo. 
Así, el análisis de diferentes tipos de materiales reunidos en colecciones puede ampliar el conocimiento acerca de un área dada o de un tema en particular. En el caso presentado en este trabajo, el análisis de los artefactos líticos de la colección Menghin tuvo como objetivo profundizar en el conocimiento acerca de la forma en que fueron explotadas las materias primas líticas representadas en los conjuntos artefactuales procedentes de la costa rionegrina del golfo San Matías, y, a su vez, evaluar la aparición o no de artefactos poco frecuentes en los sitios en la actualidad.

\section{LOS TRABAJOS INICIALES EN LA COSTA NORPATAGÓNICA Y LOS VIAJES DE O. MENGHIN}

Una de las primeras referencias de estudios en el área costera de la provincia de Río Negro la constituyen los trabajos de Félix Outes, quien menciona para el golfo la existencia de artefactos relacionados con la extracción de recursos marinos (Outes 1905). Ya en la década de 1950, y en contemporaneidad con Menghin, Leoncio Deodat realizó trabajos en la costa. A partir de sus investigaciones, este autor sugirió que, para momentos "cronológicamente remotos" (Deodat 1958-1959: 400), el golfo San Matías habría sido un lugar de periódica atracción para los indígenas. Asimismo, consideró que allí habrían habitado grupos que trabajaban las valvas de moluscos marinos y que habrían ocupado el área en vistas a alimentarse de los productos del mar (Deodat 1958-1959, 1967). Sin embargo, el autor sostiene que esta costa fue abandonada para fines del siglo XVIII, y que las rutas indígenas se trasladaron hacia tierra adentro (Deodat 1958-1959).

Oswald Menghin, catedrático de Prehistoria del Hombre y rector de la Universidad de Viena entre los años 1922 y 1945, Ilegó a la Argentina luego de la Segunda Guerra Mundial, en el año 1948. Una vez en nuestro país, fue contratado como investigador sin docencia por José Imbelloni, quien era entonces director del Museo Etnográfico de la Universidad de Buenos Aires (Mederos Martín 2014). Menghin también recibió apoyo de Claudio Sánchez-Albornoz, quien en ese momento se desempeñaba como profesor de Historia en la mencionada universidad. Pese a estos apoyos iniciales, Menghin no pudo ejercer la docencia hasta 1953, y fue en el año 1957, una vez cancelado su proceso penal en Europa, cuando obtuvo el puesto de profesor interino de
Prehistoria de la Universidad Nacional de La Plata. En ese mismo año fundó el Centro Argentino de Estudios Prehistóricos y comenzó a editar la publicación titulada Acta Præhistórica (Kohl y Pérez Gollán 2002). Finalmente, en el año 1958, le fue otorgada la cátedra de Prehistoria General y del Viejo Mundo en la Universidad de Buenos Aires, donde se jubiló en 1968 (Mederos Martín 2014).

Dentro de la Argentina, Menghin Ilevó adelante alrededor de 30 expediciones, las cuales comenzaron en la provincia de Buenos Aires. En años posteriores, realizó diversos viajes por diferentes sectores de la Patagonia, en los que siempre puso el énfasis en el trabajo de campo y en las campañas sistemáticas y prolongadas (Almagro Basch 1953-1954; Kohl y Pérez Gollán 2002; Ramundo 2012). Tanto él como en general la Escuela Histórico-Cultural de Viena, consideraban que todos los humanos tenían un origen en común y habían participado dentro del mismo proceso de creación divina universal, incluyendo a los habitantes originarios de América (Kohl y Pérez Gollán 2002). De esta manera, en todos sus trabajos aplicó los principios teórico-metodológicos de la Escuela Histórico-Cultural, la cual clasificaba los hallazgos arqueológicos en culturas, periodos y fases, formando secuencias, estilos, complejos tecnológicos, tradiciones, subtradiciones, horizontes e industrias (Politis 2003). En particular en Patagonia, Menghin explicó el desarrollo prehistórico local en relación con los planteos de los esquemas culturales del Viejo Mundo (Ramundo 2012) a partir de los restos materiales de las culturas del pasado.

El primer viaje de Menghin a la costa norpatagónica se dio en el año 1951. En este recorrido el autor visitó los concheros de San Antonio Este, de los que destacó su muy buen estado de preservación y su desconocimiento por parte de los pobladores. Esto se debería, en parte, a la ausencia de puntas de flecha, codiciadas por los coleccionistas y que solían dar lugar a la destrucción de los sitios (Menghin y Bórmida s/f: 26). A partir de estos viajes Menghin definió las industrias líticas existentes en la costa rionegrina.

Luego de estas primeras campañas, entre las décadas de 1950 y 1960 continuaron los trabajos en la costa del golfo, siempre dentro del marco de la Escuela Histórico-Cultural. Para esos momentos, Marcelo Bórmida y colaboradores localizaron alrededor de 70 sitios entre Bahía San Blas (provincia de Buenos Aires) y el arroyo Salado (provincia de Río Negro) (Bórmida 1962, 1964; Favier Dubois et al. 2008), área dentro de la cual está comprendida 
la porción de la costa rionegrina abordada en este trabajo. Estos autores correlacionaron la información geomorfológica con la artefactual para dar forma a una secuencia temporal de cambio tecnológico y cultural (Bórmida 1964). La metodología de trabajo empleada fue la definida por Menghin en los años previos (Menghin 1952), quien había propuesto la recolección y estudio de materiales en pequeñas áreas, bajo la premisa de que no era posible que se den repetidas veces las mismas condiciones de sedimentación y erosión para poder determinar a posteriori la asociación de los artefactos. Esta metodología posibilitaba, según Bórmida (1964), disponer de asociaciones reales de artefactos, que podían llegar a constituir complejos en sí mismas, pero cuya repetición permitía tener seguridad de que el investigador se hallaba frente a asociaciones culturales. De esta manera, se evitaban los errores de recolectar y mezclar diferentes complejos industriales.

Esta metodología implicaba la puesta en práctica de criterios dirigidos a separar las mínimas asociaciones primarias separables. En primer lugar, tanto Menghin como Bórmida tuvieron en cuenta la calidad de la materia prima, la presencia de núcleos y la disminución de la frecuencia en la intensidad de los hallazgos (Bórmida 1964). Otra forma de recolección consistía en el aislamiento de áreas pequeñas definidas de acuerdo con su posición en relación con las cotas de altura del terreno, y si se localizaban sobre médanos o sobre terreno firme. Finalmente, se recolectaban por separado los materiales asociados a ciertos concheros. De esta manera, Bórmida (1964) sostenía que era posible separar industrias líticas en las cuales hay asociaciones constantes de artefactos. Sobre la base de estos trabajos, entonces, el autor propuso la existencia de diferentes industrias, como por ejemplo el Jabaliense, el Puntarrubiense y el Sanmatiense, las cuales darían cuenta de la existencia en el área costera rionegrina de grupos de cazadoresrecolectores que utilizaron tecnologías simples para la explotación de recursos durante los últimos seis milenios (Bórmida 1964).

\section{ANTECEDENTES ARQUEOLÓGICOS DEL ÁREA}

La bahía de San Antonio, ubicada en el sector norte del golfo San Matías (Figura 1), pertenece a una antigua depresión litoral que fue inundada por el mar y que sufrió diversos cambios geomorfológicos desde el Pleistoceno y, en particular, desde el máximo transgresivo del Holoceno medio (Favier Dubois y Scartascini 2012). En esta área de la costa rionegrina se combinan la accesibilidad a recursos faunísticos y la presencia de agua dulce, recursos líticos y reparos topográficos (Favier Dubois y Borella 2011). La aparición conjunta de estos factores habría propiciado las visitas reiteradas a estos espacios, que se constituyeron en localidades de importancia en el área, con secuencias de ocupación extendidas en el tiempo. Así, existen en estos loci altas densidades de materiales arqueológicos, entre los que se cuentan instrumentos óseos, placas grabadas, pesas de red, puntas de proyectil, raspadores, bifaces, cáscaras de huevos decoradas, artefactos de molienda, tiestos cerámicos y enterratorios humanos (Favier Dubois et al. 2008, 2009).

De localidades situadas en la bahía de San Antonio o en sus cercanías, tales como San Antonio Este, Saco Viejo y Las Grutas, provienen los materiales de la colección Menghin depositada en el Instituto de Arqueología (IA) de la Facultad de Filosofía y Letras de la Universidad de Buenos Aires (UBA) analizados en este trabajo. La localidad Saco Viejo es una de las más conocidas de la bahía de San Antonio. Abarca un total de entre 1000 y $10.000 \mathrm{~m}^{2}$ de extensión, con numerosos asomos de concheros (Favier Dubois y Borella 2011), posee

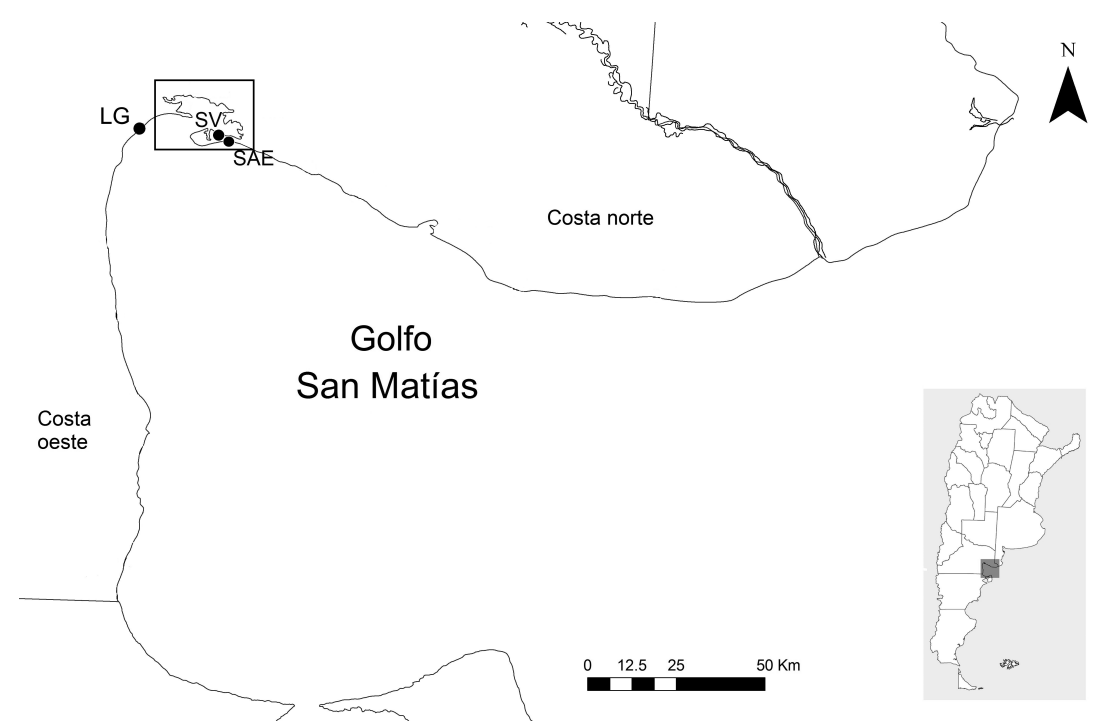

Figura 1. Golfo San Matías, provincia de Río Negro. Se señalan las localidades de donde proviene la muestra. LG: Las Grutas. SV: Saco Viejo. SAE: San Antonio Este. Dentro del recuadro se ubica la bahía de San Antonio. 
dataciones que se ubican entre los 2000 y los 400 años AP y presenta una gran diversidad y densidad artefactual (Favier Dubois et al. 2009). Análisis previos sobre diferentes materiales provenientes de esta zona expusieron la existencia de una alta frecuencia de instrumentos retocados, en particular raspadores y puntas de proyectil (Marani y Cardillo 2010). Los estudios realizados sobre estas últimas mostraron una gran variabilidad, dado que existen puntas de diferentes tamaños y formas: triangulares pedunculadas y apedunculadas de sección muy delgada y confeccionadas mediante adelgazamiento bifacial (Cardillo y Charlin 2016). Se ha propuesto que la alta frecuencia de retocadores óseos recuperados en esta localidad podría estar relacionada con la confección de estos artefactos (Marani y Cardillo 2010).

Las Grutas corresponde a una pequeña localidad arqueológica de hasta $100 \mathrm{~m}^{2}$ con lentes de concheros aisladas (Favier Dubois y Borella 2011). Allí fueron recuperados restos humanos que se fecharon entre los 2300 y los 690 años AP (García Guraieb et al. 2010).

Finalmente, en relación con la localidad de San Antonio Este, no contamos hasta el momento con información arqueológica producida por el equipo de investigación que trabaja en el área. Resta profundizar en los trabajos en esta zona particular de la bahía de San Antonio a fin de completar el panorama arqueológico de la región.

\section{METODOLOGÍA DE TRABAJO Y DESCRIPCIÓN DE LA MUESTRA ANALIZADA}

La colección Menghin estuvo en sus inicios depositada en el Museo Etnográfico "Juan B. Ambrosetti", dependiente de la Facultad de Filosofía y Letras (FFyL) de la UBA. Para los momentos en que esta colección se formó, Menghin dirigía el Centro Argentino de Estudios Prehistóricos, que estaba adherido al museo pero sin vinculación con la facultad (Fernández Distel 1985; Kohl y Pérez Gollán 2002). En el año 1973, en el marco de una reapertura del museo con una nueva política de gobierno, se realizó allí la exposición titulada "Patagonia. 12.000 años de historia". En los años previos, y durante un largo período, la institución había dado prioridad a su función como centro de investigación. Con esta exposición se comenzó con la apertura de las salas de exhibición al público en general.
Los materiales recolectados por Menghin formaron parte de dicha exhibición hasta la incorporación del Centro Argentino de Estudios Prehistóricos al entonces Instituto de Ciencias Antropológicas (ICA) de la FFyL de la UBA en el año 1974, bajo la denominación de Centro de Estudios Prehistóricos, Etnológicos y Folklóricos (Kligmann y Spengler 2016), momento en el que Bórmida mudó allí tanto los materiales de sus colecciones como los de la de Menghin. Una vez ingresados al ICA, fueron Carlos Gradin y Annette Aguerre quienes se encargaron de catalogarlos (Norma Pérez Reynoso, comunicación personal 2019). Para inicios de la década de 1980, el Centro de Estudios Prehistóricos, Etnológicos y Folklóricos pasaría a ser la Sección Prehistoria del ICA, bajo la jefatura de Amalia Sanguinetti de Bórmida, quien además ejercía como directora del instituto (Sanguinetti de Bórmida 1981).

Entre los años 1988 y 2005 las colecciones de materiales del actual IA se almacenaron en su lugar final, y en el año 2001, durante el periodo en que el Dr. Hugo Yacobaccio ejerció como director del instituto, se dio inicio al Programa de Conservación, bajo la dirección de Norma Pérez Reynoso. Desde el año 2003 la colección Menghin se encuentra almacenada en las vitrinas actuales, en 109 cajas de madera, y nueve cajas de cartón. Dentro de las cajas de madera las piezas se encuentran en las cajas de cartón y latas en las que fueron guardadas originalmente por Menghin (ver Figura 2 para algunos ejemplos). Además de los materiales de Río Negro, el conjunto está integrado también por materiales provenientes de Buenos Aires, Misiones, Santa Cruz, Córdoba, Chubut, Salta y San Juan, además de Venezuela, Paraguay, Chile, Brasil y Uruguay. No existe información certera acerca de si los artefactos provenientes del exterior fueron obtenidos por intercambio o por donación (Norma Pérez Reynoso, comunicación personal 2019). El conjunto está integrado también por materiales recolectados por Marcelo Bórmida pero que no fueron tratados en este trabajo. El hecho de que la muestra posea su catálogo propio, en el que se encuentran ingresadas todas las piezas que la integran (Figura 3), hace que la búsqueda y el acceso a los artefactos sean sencillos.

Para el estudio de la colección Menghin se emplearon los mismos criterios que se utilizan habitualmente para el análisis de las muestras arqueológicas recuperadas por el equipo de investigación. Las materias primas fueron determinadas macroscópicamente según Alberti y Fernández (2015), y la 

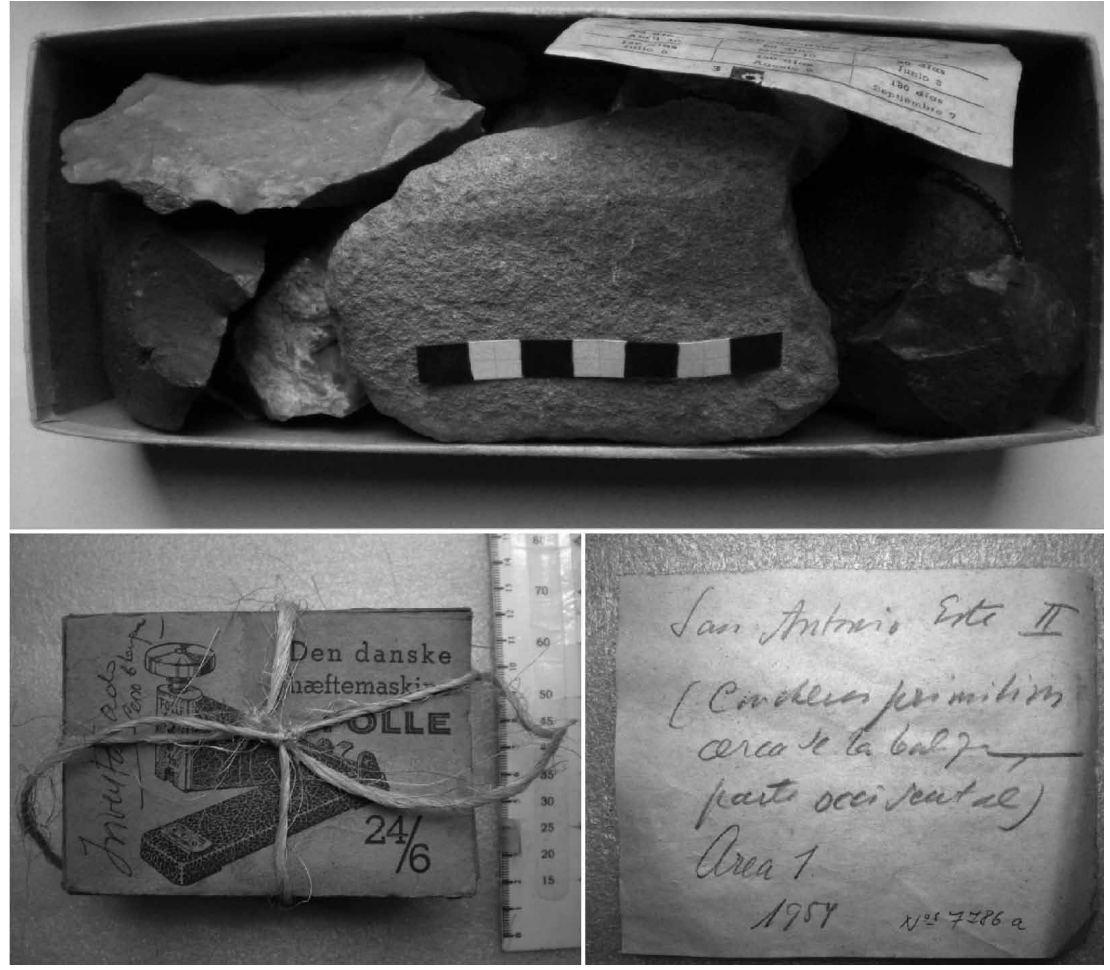

Figura 2. Detalle de las cajas de cartón originales donde se encuentran guardados los materiales y de los rótulos que las acompañan. Fotografías: Dr. M. Cardillo. calidad de las rocas para la talla según Aragón y Franco (1997). Esta clasificación comprende cuatro categorías (regular, buena, muy buena y excelente), a la que nosotros hemos agregado la de mala. Para la caracterización tecnomorfológica se siguió a Aschero $(1975,1983)$.

La muestra analizada está conformada por 398 piezas entre las que se cuentan lascas, instrumentos, núcleos, filos naturales con rastros complementarios (en adelante, FNRC) y ecofactos. La variabilidad de materias primas registradas es alta, y alcanza a 21 rocas distintas. La distribución de materiales se presenta en la Tabla 1.

El mayor porcentaje de la

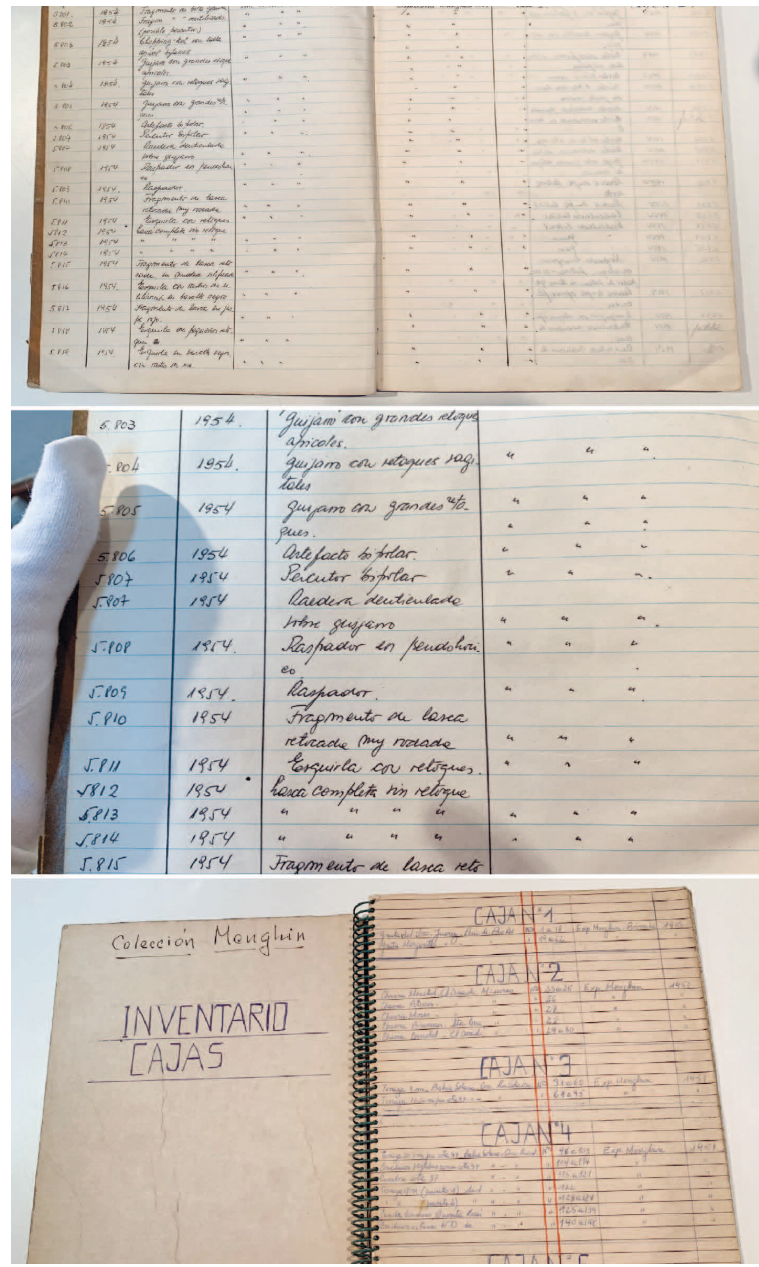

Figura 3. Catálogos de la colección O. Menghin. muestra corresponde a los instrumentos, los cuales, sumados a los FNRC, comprenden más de la mitad del conjunto (55\%). Es de destacar también la alta proporción de desechos que integran la muestra. Esta particularidad probablemente esté relacionada con la metodología aplicada por Menghin durante sus recolecciones y que fuera mencionada más arriba. Finalmente, los núcleos representan un poco más del $15 \%$ del total.

En relación con las materias primas, predomina el sílex, seguido de la calcedonia y luego las rocas volcánicas básicas. Esto continúa la tendencia ya registrada para este sector de la costa en los trabajos arqueológicos Ilevados adelante en la costa norte en general (Alberti 2016a), en los que se observó que sílex y calcedonia fueron siempre las rocas más utilizadas a nivel regional. A nivel local sí hemos detectado ciertas variaciones (ver infra). Cabe destacar la presencia de chert biogénico, que hasta el momento había aparecido de forma abundante únicamente en conjuntos de la costa oeste. En los sitios de dicha porción del golfo es una materia prima que aparece en altas proporciones (Alberti et al. 2015; Alberti 2016a, entre otros) y en diversos estadios de talla y artefactos. Debido a su abundancia, suponemos que es local de la costa oeste. Hasta el momento, su distribución había sido localizada únicamente hasta la localidad arqueológica 


\begin{tabular}{|l|c|c|c|c|c|c|c|}
\hline \multicolumn{1}{|c|}{ Materia prima } & Lascas & Núcleos & Instrumentos & FNRC & Ecofactos & $\mathbf{n}$ & $\mathbf{\%}$ \\
\hline Sílice & 53 & 35 & 75 & 12 & 1 & $\mathbf{1 7 6}$ & $\mathbf{4 4 , 2 2}$ \\
\hline Calcedonia & 21 & 2 & 44 & 6 & 0 & $\mathbf{7 3}$ & $\mathbf{1 8 , 3 4}$ \\
\hline Volcanita int.-bas. & 13 & 12 & 17 & 4 & 1 & $\mathbf{4 7}$ & $\mathbf{1 1 , 8 1}$ \\
\hline Jaspe & 2 & 6 & 8 & 1 & 0 & $\mathbf{1 7}$ & $\mathbf{4 , 2 7}$ \\
\hline Sed. silicificada & 4 & 2 & 6 & 2 & 1 & $\mathbf{1 5}$ & $\mathbf{3 , 7 7}$ \\
\hline Chert & 2 & 1 & 10 & 0 & 0 & $\mathbf{1 3}$ & $\mathbf{3 , 2 7}$ \\
\hline Ópalo & 3 & 1 & 6 & 0 & 0 & $\mathbf{1 0}$ & $\mathbf{2 , 5 1}$ \\
\hline Volcanita ácida & 3 & 0 & 4 & 0 & 2 & $\mathbf{9}$ & $\mathbf{2 , 2 6}$ \\
\hline Xilópalo & 4 & 1 & 1 & 0 & 0 & $\mathbf{6}$ & $\mathbf{1 , 5 1}$ \\
\hline Arenisca & 0 & 0 & 5 & 0 & 0 & $\mathbf{5}$ & $\mathbf{1 , 2 6}$ \\
\hline Chert biogénico & 3 & 0 & 1 & 0 & 0 & $\mathbf{4}$ & $\mathbf{1 , 0 1}$ \\
\hline Sedimentaria & 1 & 1 & 2 & 0 & 0 & $\mathbf{4}$ & $\mathbf{1 , 0 1}$ \\
\hline Cuarcita & 1 & 0 & 2 & 0 & 0 & $\mathbf{3}$ & $\mathbf{0 , 7 5}$ \\
\hline Plutonita & 0 & 0 & 3 & 0 & 0 & $\mathbf{3}$ & $\mathbf{0 , 7 5}$ \\
\hline Toba & 1 & 0 & 1 & 1 & 0 & $\mathbf{3}$ & $\mathbf{0 , 7 5}$ \\
\hline Cuarzo & 0 & 0 & 2 & 0 & 0 & $\mathbf{2}$ & $\mathbf{0 , 5}$ \\
\hline Hematita & 0 & 0 & 2 & 0 & 0 & $\mathbf{2}$ & $\mathbf{0 , 5}$ \\
\hline Obsidiana & 2 & 0 & 0 & 0 & 0 & $\mathbf{2}$ & $\mathbf{0 , 5}$ \\
\hline $\begin{array}{l}\text { Volcanita ácida } \\
\text { silicificada }\end{array}$ & 0 & 0 & 2 & 0 & 0 & $\mathbf{2}$ & $\mathbf{0 , 5}$ \\
\hline Granodiorita & 0 & 0 & 1 & 0 & 0 & $\mathbf{1}$ & $\mathbf{0 , 2 5}$ \\
\hline Toba silicificada & 0 & 0 & 1 & 0 & 0 & $\mathbf{1}$ & $\mathbf{0 , 2 5}$ \\
\hline & $\mathbf{1 1 3}$ & $\mathbf{6 1}$ & $\mathbf{1 9 3}$ & $\mathbf{2 6}$ & $\mathbf{5}$ & $\mathbf{3 9 8}$ & \\
\hline & $\mathbf{2 8 , 3 9}$ & $\mathbf{1 5 , 3 3}$ & $\mathbf{4 8 , 4 9}$ & $\mathbf{6 , 5 3}$ & $\mathbf{1 , 2 6}$ & & \\
\hline
\end{tabular}

Nota: FNRC: filos naturales con rastros complementarios. Int.-bas.: intermediabásica.

Tabla 1. Tipos y cantidad de artefactos divididos por materia prima.

de Punta Pórfido, cerca del límite con Chubut. En la costa norte no hay registro de una explotación sistemática de esta roca (Alberti 2016a).

En relación con la calidad de las materias primas que conforman la muestra analizada, la predominante es la excelente (Tabla 2), al igual que sucede en las muestras arqueológicas estudiadas (Alberti 2016a). Otro indicador utilizado fue el estado de la muestra, a partir del cual se determinó que el $69,1 \%$ de ella se encuentra entera, mientras que el $30,9 \%$ restante corresponde a artefactos fragmentados.

En relación con las categorías artefactuales, se presenta el análisis de los desechos, los núcleos, los instrumentos y los FNRC. Los desechos corresponden a lascas de arista $(43 \%, \mathrm{n}=49)$, lascas angulares $(41 \%, n=46)$, lascas secundarias $(5 \%$, $\mathrm{n}=5)$, desechos bipolares, lascas de dorso natural y hojas ( $4 \%$ cada una, $\mathrm{n}=4$ ) y solamente una

\begin{tabular}{|l|c|c|c|c|c|c|}
\hline & Lascas & Núcleos & Instrumentos & FNRC & $\mathbf{n}$ & $\%$ \\
\hline Mala & 0 & 2 & 18 & 0 & $\mathbf{2 0}$ & $\mathbf{4 , 8 3}$ \\
\hline Regular & 8 & 2 & 6 & 1 & $\mathbf{1 7}$ & $\mathbf{4 , 3 3}$ \\
\hline Buena & 19 & 5 & 21 & 3 & $\mathbf{4 8}$ & $\mathbf{1 2 , 2 1}$ \\
\hline Muy buena & 37 & 18 & 51 & 10 & $\mathbf{1 1 6}$ & $\mathbf{2 9 , 5 2}$ \\
\hline Excelente & 49 & 34 & 97 & 12 & $\mathbf{1 9 2}$ & $\mathbf{4 8 , 8 5}$ \\
\hline
\end{tabular}

Nota: FNRC: filos naturales con rastros complementarios.

Tabla 2. Cantidad y porcentaje de artefactos divididos de acuerdo con la calidad de la materia prima. lasca primaria ( $1 \%$ del conjunto). El $60 \%$ de esta muestra corresponde a desechos enteros.

Dentro del subconjunto de los núcleos, todos corresponden a núcleos de lascas. El 80,3\% del total se encuentra entero $y$, en relación con los tipos de núcleos, el $72 \%$ de la muestra corresponde a artefactos con extracciones multidireccionales, lo cual da cuenta del cambio de posición de estos durante el proceso de talla para aprovechar mejor todas las plataformas de percusión. El porcentaje restante de la muestra comprende núcleos unidireccionales e indiferenciados (26 y $2 \%$, respectivamente). Algunos ejemplos de los núcleos analizados se muestran en la Figura 4.

Dentro de los instrumentos (ver Figura 5 para algunos ejemplos), los 193 contabilizados representan un total de 204 filos manufacturados mediante percusión. Hay además 19 instrumentos manufacturados mediante abrasión, picado y pulido, $y$, finalmente, los FNRC, que ascienden a un total de 41. Entre los primeros, la mayor cantidad la constituyen los raspadores ( $\mathrm{n}=69$ filos, $33,8 \%$ ), seguidos de los denticulados ( $\mathrm{n}=30,14,7 \%$ ), las raederas $(n=17,8,33 \%)$, las preformas bifaciales $y$ los cuchillos ( $\mathrm{n}=16$ cada categoría, $7,84 \%$ ) y las muescas $(n=15,7,35 \%)$. Las puntas de proyectil constituyen un $3,9 \%$ de la muestra, con un total de ocho individuos. Este bajo número resulta interesante ya que este tipo de instrumentos suele ser objeto de recolecciones, tanto sistemáticas como asistemáticas. En esta colección en particular, puede estar asociado a la ausencia de puntas de proyectil en los concheros mencionada por Menghin y Bórmida (Menghin y Bórmida s/f). De estos grupos tipológicos, el $60 \%$ corresponde a artefactos con filos simples; el $22,75 \%$, a filos bifaciales; el $9 \%$ son artefactos con filos compuestos; y el $7 \%$ corresponde a filos dobles. Una categoría que hasta el momento no habíamos registrado en los conjuntos arqueológicos recuperados en el área son los artefactos con filos triples. Estos ascienden al $1 \%$ de la muestra, representada por un total de dos instrumentos. Aunque es un número bajo, es interesante 

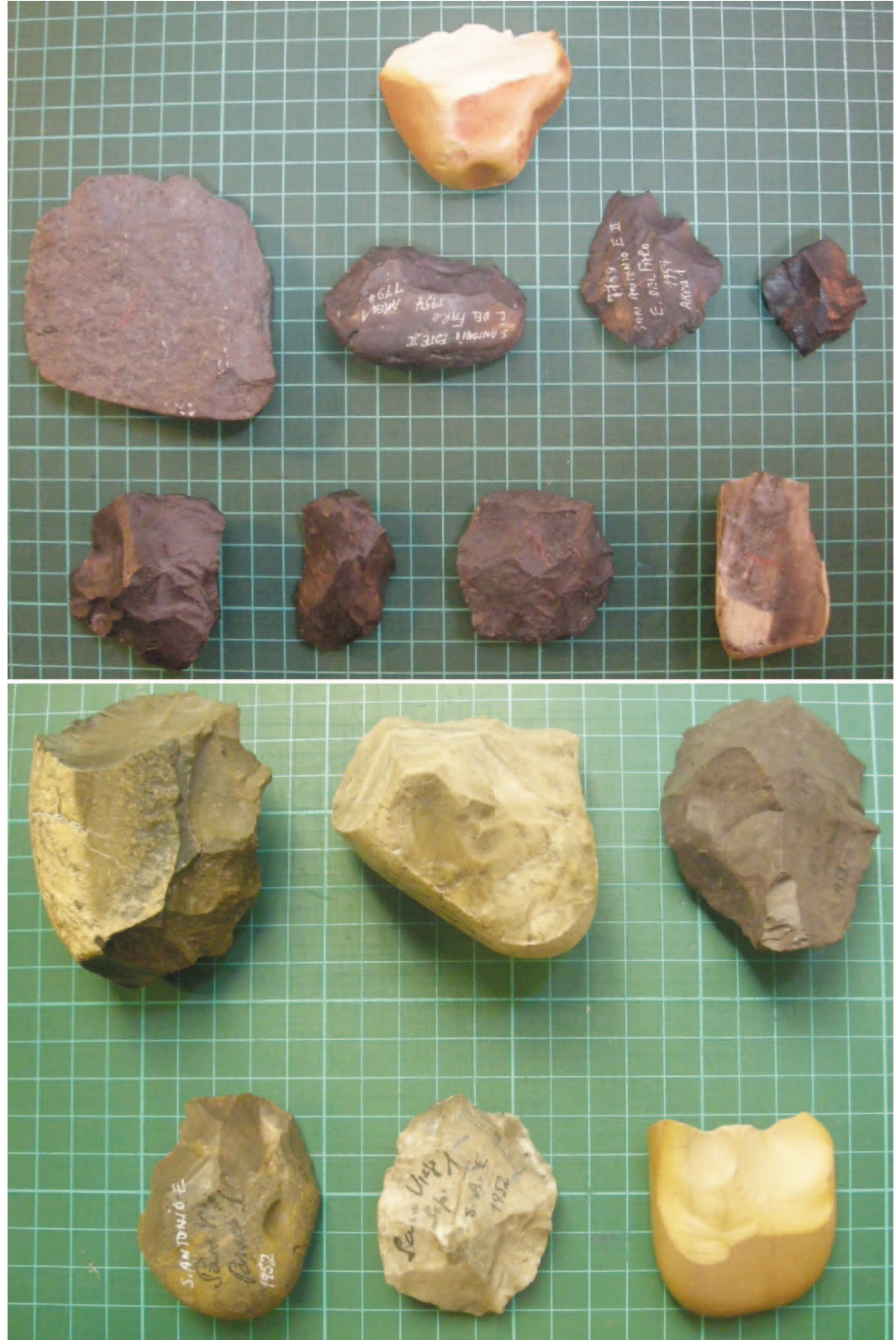

Figura 4. Núcleos integrantes de la muestra analizada. Escala: cada cuadrado de la figura representa $1 \times 1 \mathrm{~cm}$.

notar su presencia, lo cual implica pensar que estos instrumentos existieron en los conjuntos pese a que no han sido recuperados por nosotros hasta el momento.

Entre los artefactos manufacturados mediante abrasión, picado y pulido se encuentran siete bolas de boleadora, cuatro molinos, tres percutores, dos sobadores, un rompecráneo, un mortero y un yunque. El $47,3 \%$ de estos artefactos se encuentran fragmentados, mientras que el resto del conjunto está entero.

La última categoría relevada es la de los FNRC. Llama la atención la gran cantidad de este tipo de filos, que no han sido relevados con tanta frecuencia en los conjuntos de la costa norte del golfo (por ejemplo, Cardillo et al. 2007; Cardillo 2009, 2013; Cardillo y Alberti 2013; Alberti $2015 a$ y b, 2016a y b). Estos filos pueden formarse por diversas razones: por uso, por pisoteo en el campo o por el movimiento de las piezas a lo largo de los años en los depósitos. Los FNRC son mencionados en este trabajo ya que sus características (en general, negativos de lascados de tamaños relativamente uniformes y localizados de manera continua uno junto al otro) pueden haber aparecido en las piezas producto del uso. Están manufacturados principalmente sobre rocas silíceas, que provendrían de la costa oeste del golfo (Alberti 2016a), lo cual implicaría el bajo riesgo que el aprovisionamiento de materia prima lítica habría supuesto para las poblaciones que habitaron el área de la bahía de San Antonio. Es interesante destacar que en la zona, además, hay abundancia de rocas locales de calidad buena a excelente para la talla (Alberti 2016a).

\section{BREVE COMPARACIÓN CON LAS MUESTRAS ARQUEOLÓGICAS}

En estudios previos realizados sobre muestras artefactuales provenientes de Las Grutas y de Saco Viejo (Alberti 2015b, 2016b), se recuperó un total de 70 artefactos, entre los que se cuentan lascas, núcleos e instrumentos formatizados. La variabilidad de materias primas recuperadas es mucho menor que la determinada en la colección Menghin, y tal como se presenta en la Tabla 3, se destaca también el sílex en primer lugar.

En esta muestra también predominan las rocas silíceas por sobre el resto de las materias primas, aunque el segundo y el tercer lugar están ocupados por las rocas volcánicas intermedias-básicas y la calcedonia, a diferencia de lo que hemos detectado para los estudios de escala regional (Alberti 2016a). En la muestra arqueológica predominan los desechos 


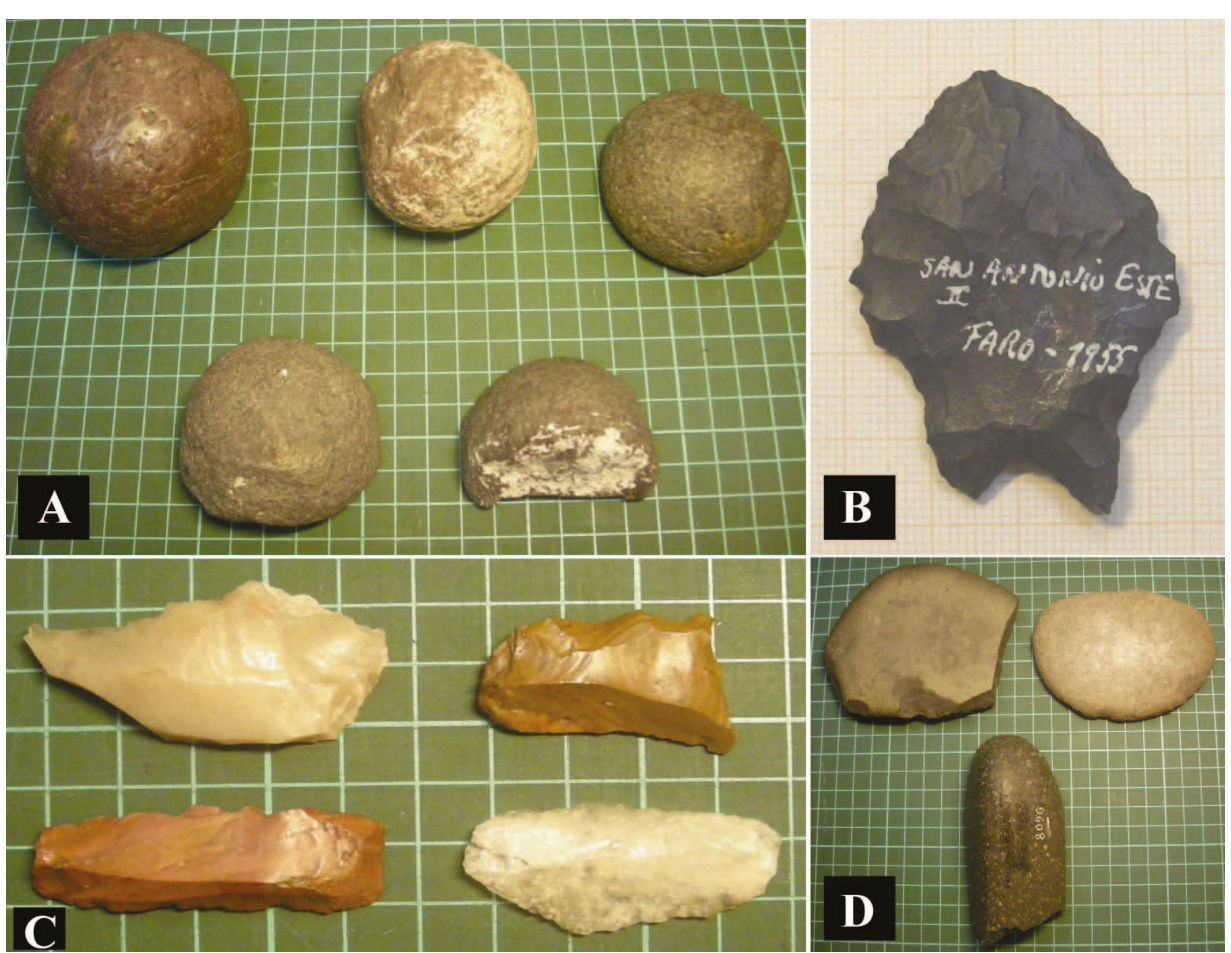

Figura 5. Instrumentos de la muestra estudiada. A) Bolas de boleadora. B) Punta de proyectil. C) Denticulados. D) Molinos y percutor. Escala: cada cuadrado de la figura representa $1 \times 1 \mathrm{~cm}$. también destacar que en las recolecciones actuales no se han recuperado filos naturales con rastros complementarios, que en los materiales de Menghin conforman un porcentaje importante. Respecto de la calidad de las rocas para la talla, también predominan la excelente y muy buena, que conforman entre las dos el $60 \%$ de la muestra analizada $(n=42)$. El porcentaje de artefactos enteros de estos conjuntos alcanza al $55,7 \%$ del total.

\begin{tabular}{|c|c|c|c|c|c|c|}
\hline Materia prima & Lascas & Núcleos & Instrumentos & Ecofactos & $\mathbf{n}$ & $\%$ \\
\hline Sílice & 17 & 2 & 3 & 0 & 22 & 31,43 \\
\hline Volcanita int.-bas. & 11 & 4 & 1 & 0 & 16 & 22,86 \\
\hline Calcedonia & 10 & 0 & 1 & 0 & 11 & 15,71 \\
\hline Chert biogénico & 4 & 1 & 0 & 1 & 6 & 8,57 \\
\hline Volcanita ácida & 5 & 0 & 0 & 0 & 5 & 7,14 \\
\hline Brecha silicificada & 2 & 0 & 0 & 0 & 2 & 2,86 \\
\hline Jaspe & 2 & 0 & 0 & 0 & 2 & 2,86 \\
\hline Ópalo & 2 & 0 & 0 & 0 & 2 & 2,86 \\
\hline Toba & 2 & 0 & 0 & 0 & 2 & 2,86 \\
\hline Sedimentaria & 0 & 0 & 1 & 0 & 1 & 1,43 \\
\hline Toba silicificada & 0 & 0 & 1 & 0 & 1 & 1,43 \\
\hline $\mathbf{n}$ & 55 & 7 & 7 & 1 & 70 & 100 \\
\hline$\%$ & 78,57 & 10 & 10 & 1,43 & & \\
\hline
\end{tabular}

Nota: Int.-bas.: intermedia-básica.

Tabla 3. Muestra arqueológica. Tipo y cantidad de artefactos divididos de acuerdo con la materia prima.

por sobre cualquier otra categoría artefactual, lo cual se repite en las muestras analizadas para la costa rionegrina del golfo en general (por ejemplo, Cardillo et al. 2007; Cardillo 2009, 2013; Cardillo y Alberti 2013; Alberti 2013, 2015a, 2016a). Es interesante también la presencia mayoritaria del chert biogénico en la muestra arqueológica con respecto a la colección Menghin, aunque siempre se mantiene en valores muy bajos en comparación con los sitios de la costa oeste (Alberti 2016a). Esto puede tener que ver con un sesgo de recolección, ya que esta materia prima se recuperó principalmente en forma de lascas, que es la categoría menos representada en la muestra de Menghin. Es importante
En relación con los tipos de desechos recuperados, en la muestra arqueológica hay mayor variabilidad de lascas, y se registran, además de los tipos mencionados en los materiales de Menghin, lascas planas y de tableta de núcleo. A diferencia de la colección, en las muestras arqueológicas no se recuperaron hojas. Dentro de los desechos, el $49 \%$ se encuentran enteros. En el grupo de los núcleos se recuperaron solo siete; todos ellos corresponden a núcleos con extracciones multidireccionales, lo que da cuenta de su cambio de posición durante el proceso de talla.
Finalmente, dentro de los instrumentos se cuentan solamente filos simples (tres raspadores y un denticulado; $57,1 \%$ ), mientras que el resto de los artefactos que integran esta categoría corresponde a una punta de proyectil, una pesa lítica y una bola de boleadora.

\section{DISCUSIÓN Y CONCLUSIONES}

El análisis de la colección Menghin permitió ampliar el panorama acerca de la tecnología lítica que manufacturaron, utilizaron y descartaron los grupos de cazadores-recolectores que habitaron 
y circularon por el golfo San Matías durante el Holoceno medio y tardío. En primer lugar, el incremento de la variabilidad de materias primas utilizadas en los instrumentos de la colección permitiría comenzar a ampliar el panorama acerca de los rangos de movilidad y circulación de estos grupos, que podría haber sido más extenso que el considerado en forma previa. Esto es particularmente importante en el caso del chert biogénico. Otra característica particular de los materiales de Menghin es que existen diferencias en las categorías de instrumentos. En la colección hay mayor número de raspadores, denticulados y artefactos manufacturados por abrasión, picado y pulido que lo que se pudo recuperar en los conjuntos arqueológicos. Esto implicaría comenzar a pensar en el procesamiento de un mayor espectro de recursos, que hasta el momento no se habían tenido en cuenta debido a la baja o nula evidencia tecnológica que pudiese ser utilizada como proxy para su abordaje. Es el caso, por ejemplo, de los recursos vegetales. Con las debidas precauciones metodológicas, es necesario comenzar a considerar, además, el caso de los FNRC que son inexistentes en los conjuntos arqueológicos. Finalmente, también hemos registrado en la colección la aparición de hojas, que en los materiales recuperados por el equipo de arqueología están ausentes. Esto comienza a abrir el panorama hacia la consideración del uso de esta tecnología en el área.

Dentro de las similitudes entre la colección y las muestras arqueológicas, podemos contar la presencia mayoritaria de artefactos enteros por sobre los fracturados, la calidad de las rocas para la talla y el alto porcentaje de núcleos de los conjuntos. En este sentido, la muestra de Menghin continúa las tendencias previamente detectadas para el área.

Las colecciones de materiales arqueológicos, recolectadas en el pasado, ya sea por viajeros, aficionados o investigadores, suelen estar integradas por artefactos enteros y de aparición poco frecuente en la actualidad en los sitios arqueológicos, y poseen una variabilidad mayor de materias primas que la determinada en las muestras recogidas por los arqueólogos. Para que el análisis de estos materiales sea efectivamente informativo y útil para la investigación, e incluso para aplicar métodos de análisis modernos, deben realizarse preguntas específicas con objetivos determinados (Petraglia y Potts 2004). Además, muchas veces estos conjuntos constituyen la única fuente de información con la que se cuenta en un área en particular y/o provienen de sitios arqueológicos que pueden haber sido destruidos, con lo que su estudio debe ser emprendido con rigurosidad metodológica, de forma tal de poder extraer toda la información posible, teniendo en cuenta las limitantes que presentan.

Consideramos que, además de ponerlas en valor científico, el estudio de colecciones de materiales arqueológicos facilita la generación de información que esté disponible tanto para la comunidad arqueológica como para el público en general, lo que las convertirá en fuentes de información útiles para la investigación. Teniendo en cuenta los objetivos de trabajo personales, pensamos además que su análisis posibilita la ampliación acerca del conocimiento de la base regional de recursos líticos a distintas escalas. Esto, en conjunto con el estudio del registro arqueológico, permitirá determinar de forma más ajustada, por ejemplo, los circuitos de movilidad, aprovisionamiento, uso y/o intercambio de materias primas en Norpatagonia.

\section{Agradecimientos}

A la Dra. Magdalena Frère, directora del Instituto de Arqueología de la Universidad de Buenos Aires, por permitir el acceso a la colección para su estudio. A la conservadora del Instituto, Norma Pérez Reynoso, por la ayuda y la información brindada para la realización de este trabajo. Al Dr. Marcelo Cardillo por cederme algunas de las fotografías que son presentadas aquí. A los Dres. Luis Borrero, Silvana Buscaglia y Leandro Luna por proporcionarme bibliografía de consulta. A los evaluadores anónimos, cuya exhaustiva revisión contribuyó a mejorar notablemente este artículo. Al Comité Editorial de la revista por la labor realizada.

\section{REFERENCIAS CITADAS}

Alberti, J.

2013 Materias primas líticas y manufactura de instrumentos en tres localidades de la costa oeste del golfo San Matías (provincia de Río Negro, Argentina). Revista Chilena de Antropología 28: 79-105.

2015a Estudio de la inversión de energía aplicada en la manufactura de instrumentos líticos recuperados en la costa rionegrina del golfo San Matías (Argentina) a lo largo del Holoceno medio y tardío. Journal of Lithic Studies 2 (2): 7-28. 
Alberti, J.

2015b Análisis de los cambios en la explotación de materias primas líticas durante el Holoceno medio y tardío en la costa norte del golfo San Matías (Río Negro, Argentina). Cuadernos de la Facultad de Humanidades y Ciencias Sociales - Universidad Nacional de Jujuy 48: 143-166.

2016a Disponibilidad y explotación de materias primas líticas en la costa de Norpatagonia (Argentina). Un enfoque regional. BAR International Series 1901, \#27. Archaeopress, Oxford.

2016b Explotación de materias primas líticas y ocupación del espacio a lo largo del Holoceno medio y tardío en la costa oeste del golfo San Matías (Río Negro, Argentina). Comechingonia 20 (2): 243-264.

Alberti, J. y V. Fernández

2015 Propuesta clasificatoria para las materias primas líticas en Patagonia (Argentina). Arqueología 21 (2): 211-235.

Alberti, J., M. Cardillo y C. Favier Dubois

2015 Fuentes de materias primas líticas en la costa del golfo San Matías (provincia de Río Negro, Argentina). Una síntesis regional. Intersecciones en Antropología. Volumen Especial 2 Materias primas líticas en Patagonia. Localización, circulación y métodos de estudio de las fuentes de rocas de la Patagonia argentino-chilena: 27-37.

Aragón, E. y N. Franco

1997 Características de rocas para la talla por percusión y propiedades petrográficas. Anales del Instituto de la Patagonia - Serie Ciencias Humanas 25: 187-199.

Alfonso-Durrruty, M., T. Bretón, T. Giles, N. Misarti, M. San Román y F. Morello

2015 Antiquity and geographic distribution of cranial modification among the prehistoric groups of FuegoPatagonia, Chile. American Journal of Physical Anthropology 158 (4): 607-623.

Almagro Basch, $M$.

1953-1954 Investigaciones del Profesor Oswaldo F. A. Menghin sobre la Prehistoria de la Argentina. Ampurias 15: 316-327.

Aschero, C.

1975 Ensayo para una clasificación morfológica de artefactos líticos aplicada a estudios tipológicos comparativos. Informe presentado al CONICET. Buenos Aires. MS.

1983 Ensayo para una clasificación morfológica de artefactos líticos. Revisión del año 1975. Informe presentado al CONICET. Buenos Aires. MS.
Balesta, B. y N. Zagorodny

2000 Memorias e intimidades de una colección arqueológica. Relaciones de la Sociedad Argentina de Antropología XXV: 41-50.

Bernal, V., P. González, I. Pérez y H. Pucciarelli 2008 Entierros humanos del noroeste de Patagonia: nuevos fechados radiocarbónicos. Magallania 36 (2): 175-183.

Bonomo, M.

2013 Reanálisis de la colección de Samuel Lothrop procedente del Delta del Paraná. Relaciones de la Sociedad Argentina de Antropología XXXVIII (1): 169-198.

Bonomo, M., I. Capdepont y A. Matarrese 2009 Alcances en el estudio de colecciones. Los materiales arqueológicos del delta del río Paraná depositados en el museo de La Plata (Argentina). En Arqueología Suramericana, Vol. 5 (1), editado por C. Gnecco y A. Haber, pp. 68-101. Universidad Nacional de Catamarca, Catamarca.

Borella, F. y N. Buc

2009 Ópticas y ópticos. Una aproximación a la tecnología ósea en la Bahía de San Antonio (Río Negro), Argentina. En Arqueología de la Patagonia. Una mirada desde el último confín, editado por M. Salemme, F. Santiago, M. Álvarez, E. Piana, M. Vázquez y E. Mansur, pp. 421-432. Utopías, Ushuaia.

Bórmida, $M$.

1962 El Jabaliense: una industria de guijarros de la península de San Blas, provincia de Buenos Aires (República Argentina). Trabajos de Prehistoria VI. Instituto Español de Prehistoria, Madrid.

1964 Arqueología de la costa norpatagónica. Trabajos de Prehistoria XV. Instituto Español de Prehistoria, Madrid.

Borrero, L.

2015 Con lo mínimo: los debates sobre el poblamiento de América del Sur. Intersecciones en Antropología 16: 5-38.

Borrero, L. y F. Borella 2010 Harpoons and travellers: Fuegian ethnographic collections and the recent archaeological record. Before Farming 3: 1-14.

Buc, N. y V. Coronel 2013 Revisando la colección de instrumentos óseos de L. M. Torres (D25 Museo de Ciencias Naturales de La Plata). Arqueología 19 (2): 245-264. 
Cardillo, M.

2009 Variabilidad en la manufactura y diseño de artefactos en el área costera patagónica. Un enfoque integrador. Tesis Doctoral inédita. Facultad de Filosofía y Letras, Universidad de Buenos Aires, Buenos Aires. 2013 Diversidad y distribución de los conjuntos líticos asignables al Holoceno medio-tardío en la costa norte del Golfo San Matías. Provincia de Río Negro. Argentina. En Tendencias teórico-metodológicas y casos de estudio en la arqueología de la Patagonia, compilado por A. F. Zangrando, R. Barberena, A. Gil, G. Neme, M. Giardina, L. Luna, C. Otaola, S. Paulides, L. Salgán y A. Tivoli, pp. 63-69. Museo de Historia Natural de San Rafael, San Rafael.

Cardillo, M. y J. Alberti

2013 Diversidad en el instrumental lítico y uso del espacio durante el Holoceno medio y tardío en la costa norte del golfo San Matías (Río Negro, Argentina). Magallania 41 (1): 241-253.

Cardillo, M. y J. Charlin

2016 Morphological Diversification of Stemmed Projectile Points of Patagonia (Southernmost South America). Assessing Spatial Patterns by Means of Phylogenies and Comparative Methods. En Multidisciplinary Approaches to the Study of Stone Age Weaponry, editado por E. Delson y E. Sargis, pp. 261272. Vertebrate Paleobiology and Paleoanthropology Book Series, Springer, Alemania.

Cardillo, M., C. Favier Dubois, F. Scartascini y L. Lípari 2007 Una aproximación a la diversidad en la tecnología lítica de la costa Norte del Golfo San Matías, Río Negro, Argentina. Tras las huellas de la materialidad. Actas del XVI Congreso Nacional de Arqueología Argentina, pp. 323-328. San Salvador de Jujuy.

Castro Esnal. A.

2015 "Tropezando con las mismas piedras". Las colecciones de material lítico de la Patagonia argentina en el Museo de América. Anales del Museo de América XXIII: 32-47.

Deodat, L.

1958-1959 El golfo San Matías y las veredas indígenas rionegrinas. Runa 1-2: 391-404.

1967 Una antigua manufactura valvácea en el golfo de San Matías (Argentina). Runa 10 (1-2): 319-353.

Favier Dubois, C. y F. Borella

2011 Contrastes en la costa del golfo: una aproximación al estudio del uso humano del litoral rionegrino. En Arqueología de pescadores y marisqueadores en Nordpatagonia. Descifrando un registro de más de
6.000 años, editado por F. Borella y M. Cardillo, pp. 13-42. Dunken, Buenos Aires.

Favier Dubois, C., F. Borella, L. Manzi, M. Cardillo, S. Lanzellotti, F. Scartascini, M. Carolina y E. Borges Vaz 2008 Aproximación regional al registro arqueológico de la costa rionegrina. En Arqueología de la Costa Patagónica. Perspectivas para la conservación, editado por I. Cruz y S. Caracotche, pp. 50-68. Universidad Nacional de la Patagonia Austral, Río Gallegos.

Favier Dubois, C., F. Borella y R. Tykot 2009 Explorando tendencias en el uso humano del espacio y los recursos en el litoral rionegrino (Argentina) durante el Holoceno medio y tardío. En Arqueología de la Patagonia. Una mirada desde el último confín, editado por M. Salemme, F. Santiago, M. Álvarez, E. Piana, M. Vázquez y E. Mansur, pp. 985997. Utopías, Ushuaia.

Favier Dubois, C. y F. Scartascini

2012 Intensive fishery scenarios on the North Patagonian coast (Río Negro, Argentina) during the Mid-Holocene. Quaternary International 256: 62-70.

Fernández Distel, A.

1985 Prehistoria. En Evolución de las Ciencias en la República Argentina. 1872-1972. Tomo X: Antropología, pp. 83-104. Sociedad Científica Argentina, Buenos Aires.

Ford, R.

1979 Paleoethnobotany in American Archaeology. En Advances in archaeological method and theory 2, editado por M. Schiffer, pp. 285-226. Academic Press, Nueva York.

García Guraieb, S., C. Mariano y C. Favier Dubois 2010 El Buque Sur: un entierro primario múltiple de 2300 años en la costa del golfo San Matías, Río Negro, Argentina. Magallania 38 (1): 137-148.

Goetze, C. y B. Mills

1991 An assessment of the research potential of Museum collections: the Babbit Collection at the Museum of Northern Arizona. Kiva 57 (1): 77-91.

Kligmann, D. y G. Spengler 2016 Análisis histórico de una publicación científica especializada: pasado, presente y futuro de la Revista Arqueología a 25 años de su creación. Arqueología 22 (1): 15-60.

Kohl, P. y J. Pérez Gollán 2002 Religion, politics, and prehistory. Reassessing the lingering legacy of Oswald Menghin. Current Anthropology 43 (4): 561-586. 


\section{Marani, H y M. Cardillo}

2010 Retocadores óseos de Saco Viejo (Río Negro, Argentina). Un enfoque morfogeométrico. En Zooarqueología a principios del siglo XXI: aportes teóricos, metodológicos y casos de estudio, editado por M. Gutiérrez, M. de Nigris, P. Fernández, M. Giardina, A. Gil, A. Izeta, G. Neme y H. D. Yacobaccio, pp. 453-458. Del Espinillo, Buenos Aires.

Mederos Martín, A.

2014 El espejismo nacional-socialista. La relación entre dos catedráticos de Prehistoria, Oswald Menghin y Julio Martínez Santa-Olalla (1935-1952). Trabajos de Prehistoria 71: 199-220.

Menghin, O.

1952 Fundamentos cronológicos de la prehistoria de Patagonia. Runa 5: 23-43.

Menghin, O. y M. Bórmida

s/f Arqueología de la costa patagónica. MS.

Outes, F.

1905 La Edad de Piedra en Patagonia. Anales del Museo Nacional 12: 203-574.

Parezo, N.

1987 The information of ethnographic collections: The Smithsonian Institution in the American Southwest.

Advances 10: 1-47.
Pérez de Micou, C.

1998 Las colecciones arqueológicas y la investigación. Revista do Museu de Arqueologia e Etnologia 8: 223-233.

Petraglia, M. y R. Potts 2004 The Old World Paleolithic and the development of a national collection, Washington, D.C. Smithsonian Contributions to Anthropology Series 48, Washington.

Politis, G.

2003 The theoretical landscape and the methodological development of archaeology in Latin America. Latin American Antiquity 14 (2): 115-142.

Ramundo, P.

2012 Arqueología argentina: Pampa y Patagonia en perspectiva histórica. Atek Na 2: 75-120.

Sanguinetti de Bórmida, A. 1981 Palabras preliminares. Trabajos de Prehistoria 1: 9-11.

Zilio, L., S. Buus y H. Hammond 2018 La colección arqueológica "Pedro Dade" del Museo de La Plata. Revista del Museo de La Plata 3 (2): 368-392. 
